\title{
Práticas de educação em saúde realizadas por enfermeiros para pacientes do programa hiperdia
}

Health education practices carried out by nursesfor patients of hiperdia program

Practicas de educación en salud realizadas por enfermeirospara pacientes del programa hiperdia

\author{
Sílvio NICOLAU ${ }^{(1)}$ \\ Kelly Joelma Dantas BATISTA ${ }^{(2)}$ \\ Adriana MOURA ${ }^{(2)}$ \\ Jackeline SIMAS ${ }^{(2)}$ \\ (1) Universidade Federal de Pernambuco - UFPE, Departamento de Enfermagem, Recife, PE, Brasil. \\ (2) Faculdade Mauricio de Nassau - UNINASSAU, Curso de Enfermagem, Recife, PE, Brasil.
}

Recebido:14 fev 2017 Revisado: 14 out 2017 Aceito: 23 nov 2017

\section{Autor de}

correspondência: Sílvio Nicolau

silvio.nicolau@gmail.com

Conflito de interesses: Os autores declaram não haver nenhum interesse profissional ou pessoal que possa gerar conflito de interesses em relação a este manuscrito.

\section{Resumo}

Introdução: Um dos temas mais relevantes, no que diz respeito aos agravos à saúde, e para qual o trabalho da educação em saúde é de extrema importância, refere-se à Hipertensão Arterial Sistêmica e ao Diabetes Mellitus. Objetivo: identificar as características da produção nacional sobre as práticas de educação em saúde realizadas por enfermeiros para pacientes nos programas de doenças crônicas para a hipertensão arterial sistêmica e o diabetes mellitus. Método: trata-se de uma Revisão Integrativa, de cunho descritivo, realizado utilizando como fontes de pesquisa as bases de dados ScIELO, LILACS e BDEnf, amostra resultou em 15 artigos após a utilização dos critérios de inclusão e exclusão, incluindo estudos publicados entre os anos de 2011 e 2016, para coleta de dados foi utilizado instrumento elaborado pelo pesquisador. Resultados: Decorrente da apreciação dos estudos elencados foi possível categorizar os achados em dois eixos temáticos, a saber, Metodologia Freireana utilizando grupos de educação em saúde, como também Métodos tradicionais e a utilização das palestras educativas. Considerações Finais: De acordo com os dados coletados durante a pesquisa, notou-se que a literatura aponta uma tendência de seguir os moldes tradicionais, evidenciados no percentual de $40 \%$ com as palestras educativas, conforme consta nos artigos e periódicos pesquisados.

Descritores: Educação em Saúde; Enfermagem; Hipertensão; Diabetes Mellitus. 


\begin{abstract}
Introduction: One of the most relevant issues with regard to health problems, and for which the work of health education is extremely important, refers to Systemic Arterial Hypertension and Diabetes Mellitus. Objective: to identify the characteristics of the national production on the health education practices performed by nurses for patients in the programs of chronic diseases for systemic arterial hypertension and diabetes mellitus. Method: This is a descriptive Integrative Review, carried out using the SCIELO, LILACS and BDEnf databases as a research source; the sample resulted in 15 articles after the inclusion and exclusion criteria were used, including studies published among the Years of 2011 and 2016, for data collection was used instrument elaborated by the researcher. Results: based on the appreciation of the studies listed, it was possible to categorize the findings into two thematic axes, namely Freirean Methodology using health education groups, as well as traditional methods and the use of educational lectures. Final Considerations: According to the data collected during the research, it was noticed that the literature indicates a tendency to follow the traditional molds, evidenced in the percentage of $40 \%$ with the educational lectures, as it appears in the articles and periodicals researched.
\end{abstract}

Keywords: Health Education; Nursing; Hypertension; Diabetes Mellitus.

\begin{abstract}
Resumen
Introducción: Uno de los temas más relevantes, en lo que se refiere a los agravios a la salud, y para el cual el trabajo de la educación en salud es de extrema importancia, se refiere a la Hipertensión Arterial Sistémica y la Diabetes Mellitus. Objetivo: identificar las características de la producción nacional sobre las prácticas de educación en salud realizadas por enfermeros para pacientes en los programas de enfermedades crónicas para la hipertensión arterial sistémica y la diabetes mellitus. Método: se trata de una Revisión Integrativa, de cuño descriptivo, realizado utilizando como fuentes de investigación las bases de datos SCIELO, LILACS y BDEnf, muestra resultó en 15 artículos después de la utilización de los criterios de inclusión y exclusión, incluyendo estudios publicados entre los años de 2011 y 2016, para la recolección de datos fue utilizado instrumento elaborado por el investigador. Resultados: Después de la apreciación de los estudios enumerados fue posible categorizar los resultados en dos ejes temáticos, a saber, Metodología Freireana utilizando grupos de educación en salud, así como Métodos tradicionales y la utilización de las conferencias educativas. Consideraciones finales: De acuerdo con los datos recolectados durante la investigación, se notó que la literatura apunta una tendencia a seguir los moldes tradicionales, evidenciados en el porcentaje del $40 \%$ con las charlas educativas, conforme consta en los artículos y periódicos encuestados.
\end{abstract}

Palabras-claves: Educación en Salud; Enfermería; Hipertensión; Diabetes Mellitus.

\title{
Introdução
}

A educação em saúde é ferramenta de extrema importância para a promoção da saúde pública. No início do século $X X$, o foco do Governo estava preocupado nas epidemias como a varíola, a malária, a febre amarela, e posteriormente a peste. Nas escolas, reduzia-se a ensinamentos de higiene pessoal, sem levar em consideração as alterações comportamentais que a sociedade estava enfrentando. A importância da saúde 
pública no aspecto educacional ganhou novos modelos com a incorporação das diretrizes promovidas com a publicação da Carta de Ottawa, na qual se afirma que a promoção de saúde é fornecer às populações opções para que sejam capazes de melhorar sua saúde e exercer controle sobre ela. ${ }^{1,2}$

Desta forma, é possível afirmar que as práticas aplicadas eram dirigidas pelas concepções de saúde e de educação em saúde vivenciadas em cada momento da história e pelos moldes de atenção à saúde.Outro fato importante que marca o contexto de reorganização das práticas educativas foi a Conferência Internacional sobre cuidados primários de saúde, realizada em Alma-Ata no ano de 1978. Esta conferência indica que era necessária a elaboração de ações capazes de dar conta da diversidade da população, e na oferta de métodos que busquem, simultaneamente, uma forma de conceito e metodologia no desenvolvimento das práticas de saúde pública, que incluam ações educacionais. $^{3,4}$

Sabe-se que as manifestações sociais, científicas e políticas sempre foram molas propulsoras, agentes de mudança e evolução da humanidade como um todo. Diante dos erros e acertos que marcaram o desenvolvimento da área da saúde, torna-se notoriamente importante buscar meios para que se possa assegurar uma melhor qualidade de vida - QV para as pessoas, enfatizando as práticas de saúde coletiva. No entanto, vale ressaltar que as definições de educação e promoção da saúde são consideradas paradigmas, entende-se por educação em saúde quaisquer combinações de experiências de aprendizagem delineadas com vistas a facilitar ações voluntárias conducentes à saúde. ${ }^{5,6}$

Um dos temas mais relevantes, no que diz respeito aos agravos à saúde, e para qual o trabalho da educação em saúde é de extrema importância, refere-se à hipertensão arterial sistêmica - HAS e ao diabetes mellitus- DM. A HAS está relacionada com a força de impulsão que o coração exerce para levar o sangue para o resto do corpo. Entretanto, para ser considerado hipertenso, é preciso que o indivíduo permaneça com a pressão elevada por um período prolongado de tempo. O DM é uma doença do metabolismo que provoca 
a disfunção na produção de insulina, tendo como característica o aumento da glicose no sangue, levando o paciente a complicações crônicas. ${ }^{7,8}$

Ambas doenças possuem agravos semelhantes, como Infarto Agudo do Miocárdio - IAM, Acidente Vascular Cerebral - AVC e doenças renais. Os fatores de risco mais recorrentes são o tabagismo, sedentarismo, obesidade e histórico familiar de Doenças Cardiovasculares - DCV. Tais enfermidades tornam-se uma das maiores causas nos números de óbitos, internações hospitalares e amputações dos membros inferiores, no caso da DM, como uma das consequências de suas variadas complicações. ${ }^{8,9}$

A HAS e o DM são Doenças Crônicas Não Transmissíveis - DCNT, com um índice de prevalência elevado, de alto custeio social e de enorme impacto na mortalidade da população mundial. Para combater esses dados alarmantes, o Governo procurou implementar intervenções no âmbito da saúde pública, tratando a problemática de uma maneira mais organizada e eficaz, criando em 2001 o Plano de Reorganização da Atenção à Hipertensão Arterial e ao Diabetes Mellitus- HIPERDIA. O objetivo desse programa consiste em organizar a assistência no âmbito da Atenção Primária à Saúde - APS, por meio da atualização e capacitação da equipe de saúde. ${ }^{10,11}$

Percebe-se, que o campo para o desenvolvimento das práticas educativas de enfermagem aos portadores de HAS e DM encontra-se inserido na Estratégia de Saúde da Família - ESF, definida como o conjunto de ações da APS, voltada para a prevenção dessas doenças. Os desafios do controle e prevenção da HAS e DM são desenvolvidos pelas equipes de Atenção Básica - $A B$, na qual o enfermeiro exerce seu papel profissional, juntamente com uma equipe de saúde, a qual o desenvolvimento do trabalho implica uma ligação com a comunidade, levando em consideração a diversidade socioeconômica. ${ }^{12,13}$

$\mathrm{Na} E S F$, o atendimento ao paciente é multiprofissional e interdisciplinar, cada profissional realiza seu atendimento, e em equipe, traçam suas respectivas metas, de modo a desenvolver as intervenções necessárias para a manutenção e recuperação da saúde da população. A consulta de enfermagem é direcionada às ações educativas e tem como 
objetivo incentivar as pessoas a terem uma devida atenção com os cuidados à sua saúde, e leva ao paciente e à população as informações e os esclarecimentos referentes à suas respectivas enfermidades, com incentivo a mudança de estilo de vida. ${ }^{14,13}$

Vale ressaltar que este estudo não representa a totalidade do assunto, como também está direcionado para a perspectiva do preenchimento de lacunas no conhecimento. Diante dos argumentos expostos, esta pesquisa tem como objetivo, identificar as características da produção nacional sobre as práticas de educação em saúde realizadas por enfermeiros para pacientes do programa HIPERDIA, descrevendo as práticas educativas aplicadas que aparecem com maior frequência nos artigos e periódicos utilizados.

\section{Metodologia}

Trata-se de um estudo descritivo, exploratório, com suporte em uma Revisão Integrativa (RI) da literatura. Este tipo de revisão contribui para o processo de sistematização e análise dos resultados relacionados ao referido tema. A RI tem como finalidade compreender determinado tema, como também é a mais ampla abordagem metodológica referente às revisões, permitindo a inclusão de estudos experimentais e não experimentais para uma melhor compreensão do fenômeno analisado o presente estudo tem como ponto de partida estudos relacionados à educação em saúde. ${ }^{15,16}$

A escolha dessa metodologia possibilita incluir dados da literatura teórica e empírica. Inicialmente, é necessário seguir um protocolo desenvolvido do estudo de Whittemore ${ }^{16}$ com as seis etapas envolvidas durante o processo e realização da pesquisa. Desta forma as seis etapas indicadas para a constituição da revisão integrativa da literatura estão representadas na Figura 1. 


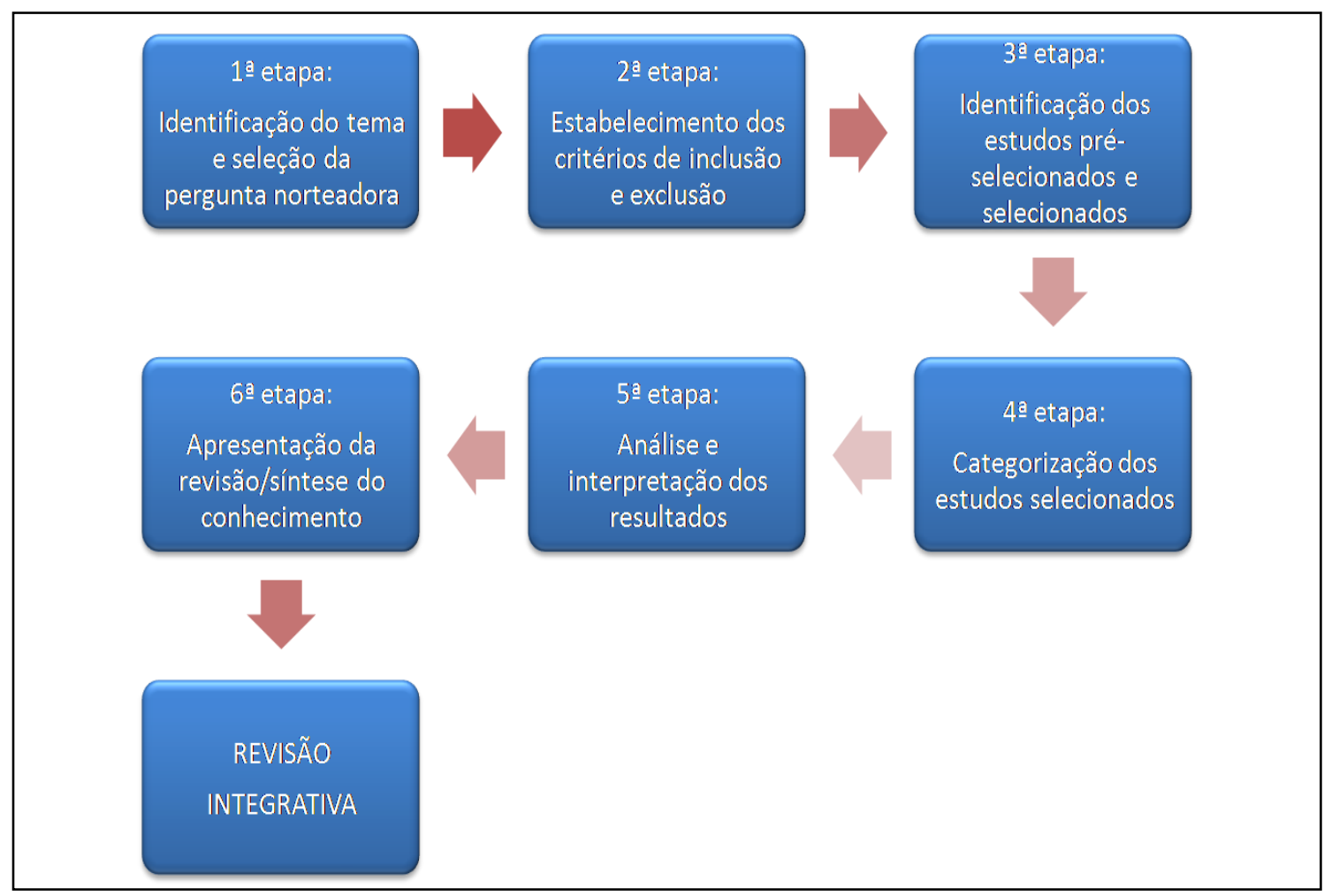

Figura 1. Diagrama de representação das seis etapas para a realização da Revisão Integrativa (RI). Recife-PE. 2017.

Fonte: Elaboração própria (2016).

O estudo foi realizado no segundo semestre de 2016, de maneira sistematizada, adotando-se a abordagem bibliográfica, buscando responder a pergunta norteadora da pesquisa: "quais as características da produção nacional sobre as práticas de educação em saúde realizadas por enfermeiros para pacientes do programa HIPERDIA?"

Foram utilizados como critérios de inclusão, trabalhos relacionados a seres humanos, artigos completos e do tipo original, artigo de reflexão, estudo de caso ou relato de experiência. Foram utilizados também como delimitação da pesquisa, artigos publicados entre os anos de 2011 a 2016, publicados em língua portuguesa, e em periódicos nacionais. Como critérios de exclusão, foram levados em consideração os artigos publicados no formato de revisão, para dar maior poder de evidencia ao estudo em desenvolvimento, como também aqueles encontrados em duplicidade. 
Para realizar a busca, foram selecionadas e consultadas a ScientificElectronic Library Online SciELO, Literatura Latino-Americana e do Caribe em Ciências da Saúde - LILACS e Base de Dados de Enfermagem - BDEnf. Para ampliar o potencial de busca e escolha da amostragem, foi processado o Cruzamento por Descritores - CD, conforme a lista constante nos Descritores em Ciência da Saúde (DeCS). No cruzamento por descritores, foi utilizada a estratégia de busca associada com o operador booleano "AND", configurado como "Educação em Saúde" AND "Enfermagem"; "Educação em Saúde" AND "Diabetes Mellitus"; "Educação em Saúde" AND "Hipertensão"; A pesquisa foi realizada no período de Julho a Agosto de 2016, levando em consideração os critérios de pesquisa supracitados.

Desta forma, obteve-se a amostra pertinente para o tipo de estudo e com o quantitativo de estudos necessários para organizar os resultados e elaborar as discussões, conforme apresentado no Quadro 1.

\begin{tabular}{|l|c|c|c|c|c|c|c|c|}
\hline \multicolumn{8}{|c|}{ Principais fontes de pesquisa utilizadas na coleta de dados } \\
\hline \multirow{2}{*}{ Descritores } & \multicolumn{2}{|c|}{ SciELO } & \multicolumn{2}{c|}{ LILACS } & \multicolumn{2}{c|}{ BDENF } & \multicolumn{2}{c|}{ TOTAL } \\
\cline { 2 - 9 } & $\mathbf{n}$ & $\mathbf{\%}$ & $\mathbf{n}$ & $\mathbf{\%}$ & $\mathbf{n}$ & $\%$ & $\mathbf{n}$ & $\%$ \\
\hline Diabetes Mellitus & 1.739 & 3,01 & 790 & 1,36 & 533 & 0,93 & 3.062 & 5,30 \\
\hline Hipertensão & 2.751 & 4,76 & 1.284 & 2,22 & 637 & 1,11 & 4.672 & 8,09 \\
\hline Educação em Saúde & 3.884 & 6,73 & 5.465 & 9,47 & 3.788 & 6,57 & 13.137 & 22,77 \\
\hline Enfermagem & 13.107 & 22,74 & 4.305 & 7,48 & 19.398 & 33,62 & 36.810 & 63,84 \\
\hline Total & $\mathbf{2 1 . 4 8 1}$ & $\mathbf{3 7 , 2 4}$ & $\mathbf{1 1 . 8 4 4}$ & $\mathbf{2 0 , 5 3}$ & $\mathbf{2 4 . 3 5 6}$ & $\mathbf{4 2 , 2 3}$ & $\mathbf{5 7 . 6 8 1}$ & $\mathbf{1 0 0 , 0}$ \\
\hline
\end{tabular}

Quadro 1. Distribuição quantitativa da amostragem conforme os descritores e o banco de dados. Recife-PE, 2016.

Fonte: Elaboração própria (2016).

A partir do material obtido, foi realizada uma leitura criteriosa de cada resumo, destacando aqueles que respondem ao objetivo proposto por este estudo e o tema relacionado à educação em saúde realizada por enfermeiros no programa HIPERDIA. Para a organização dos dados, foi elaborado pelos autores da pesquisa um formulário como instrumento de coleta de dados contendo títulos, periódicos, ano da publicação, o perfil 
profissional dos autores e a distribuição por categoria do estudo, assim como também as estratégias educativas realizadas pelos profissionais de enfermagem.

A avaliação crítica, análise e coleta de dados, foram obtidas por meio de um formulário, sendo este utilizado como guia metodológico, conforme dito anteriormente, assim como também a avaliação crítica dos estudos, na qual foi observada a pertinência do rigor metodológico escolhido em relação ao objeto de investigação.

Após análise dos critérios de inclusão e exclusão de acordo com o número de artigos encontrados, deu-se a apreciação bibliográfica para categorização e organização dos estudos selecionados. Decorrente da apreciação dos estudos elencados foi possível categorizar os achados em dois eixos temáticos, a saber, Metodologia Freireana utilizando grupos de educação em saúde, como também Métodos tradicionais e a utilização das palestras educativas.

Para maior embasamento teórico e aprofundamento na discussão, foram utilizados outros artigos, teses de doutorado, decretos do Ministério da Saúde - MS, como também pesquisas relacionadas a Políticas de Saúde Pública. Para a realização do processamento dos dados, as informações que foram coletadas em planilhas, como também os resultados foram organizados por meio do programa Excel ${ }^{\circledR}$ do Windows, para criação e formatação de gráficos e quadros. Esta etapa permitiu a avaliação e comparação dos estudos, categorizando os resultados, e por fim, a síntese dos resultados é representada pela síntese das categorias.

Quanto aos aspectos éticos do estudo, respeitou-se a autoria de todos os artigos estudados. Vale ressaltar que os estudos revisados foram geralmente pequenos, isso pode ter limitado a generalização dos achados, a análise de subgrupos foi realizada em todo o conjunto de estudos. 


\section{Resultados}

Os dados deste estudo apresentam-se de forma a facilitar o entendimento e a discussão dos mesmos, para melhor visualização os resultados estão apresentados na forma de gráficos e quadros. O presente estudo encontrou uma quantidade significativa de artigos científicos conforme as bases de dados de origem, sendo estas a SciELO, Lilacs e BDEnf, relacionados com os descritores em cada pesquisa.

De acordo com a amostragem foram encontrados 21 artigos de relevância sobre educação em saúde realizada pelo enfermeiro, que após uma análise criteriosa, viu-se que dentre estes artigos, 15 deles demonstraram relevância e foram usados como objeto de pesquisa do presente estudo.

\begin{tabular}{|c|c|c|c|}
\hline \multicolumn{4}{|c|}{ Artigos selecionados relacionados às bases de dados e descritores utilizados } \\
\hline \multirow{2}{*}{ Bases } & Descritores & AE & AS \\
\hline \multirow{2}{*}{ SCIELO } & Educação em saúde AND Hipertensão & 77 & 4 \\
\cline { 2 - 4 } & Educação em saúde AND Diabete Mellitus & 90 & 3 \\
\hline \multirow{2}{*}{ LILACS } & Educação em saúde AND Enfermagem & 81 & 2 \\
\cline { 2 - 4 } & Educação em saúde AND Hipertensão & 142 & 2 \\
\hline \multirow{2}{*}{ BDENF } & Educação em saúde AND Diabetes Mellitus & 165 & 2 \\
\cline { 2 - 4 } & Educação em saúde AND Hipertensão & 137 & 2 \\
\hline
\end{tabular}

Quadro 2. Distribuição quantitativa da amostragem após a utilização dos operadores booleanos, Recife-PE, 2016.

Fonte: Elaboração própria (2016).

$\mathrm{AE}=$ Artigos Encontrados; $\mathrm{AS}=$ Artigos Selecionados.

Neste sentido, foram utilizadas as pesquisas que estiveram em maior concordância com o tema, que continham uma abordagem descritiva sobre as intervenções de enfermagem para educação em saúde, excluindo os demais que não se encaixavam com a temática abordada, as quais estão apresentadas no Quadro 3. 
ISSN 2179-6750

Características da amostra quanto às práticas de educação em saúde realizadas pelo enfermeiro

\begin{tabular}{|c|c|c|c|c|}
\hline $\mathrm{N}^{\mathrm{O}}$ & Título & Ano & Titulação & Intervenções de Enfermagem \\
\hline 1 & $\begin{array}{c}\text { Educação em saúde como melhoria da qualidade } \\
\text { de vida dos usuários hipertensos }\end{array}$ & 2011 & Graduando & Palestras educativas \\
\hline 2 & $\begin{array}{c}\text { Visita domiciliar: estratégia educativa para o } \\
\text { autocuidado de clientes diabéticos na atenção } \\
\text { básica }\end{array}$ & 2011 & Mestre & Visita domiciliar \\
\hline 3 & $\begin{array}{c}\text { Hiperdia: adesão e percepção de usuários } \\
\text { acompanhados pela estratégia de saúde da } \\
\text { família }\end{array}$ & 2011 & Especialista & Palestras educativas \\
\hline 4 & $\begin{array}{l}\text { O cuidado na visão de portadores de hipertensão } \\
\text { arterial }\end{array}$ & 2012 & Especialista & $\begin{array}{l}\text { Grupos de educação em } \\
\text { saúde }\end{array}$ \\
\hline 5 & $\begin{array}{l}\text { Educação em saúde no trabalho de enfermeiras } \\
\text { em Santarém do Pará, Brasil }\end{array}$ & 2012 & $\begin{array}{c}\text { Não } \\
\text { divulgado }\end{array}$ & $\begin{array}{l}\text { Grupos de educação em } \\
\text { saúde }\end{array}$ \\
\hline 6 & $\begin{array}{l}\text { Práticas educativas em diabetes mellitus: } \\
\text { compreendendo as competências dos } \\
\text { profissionais da saúde }\end{array}$ & 2012 & Mestre & Atividades educativas \\
\hline 7 & $\begin{array}{c}\text { Percepção dos profissionais de saúde sobre o } \\
\text { trabalho interdisciplinar nas estratégias educativas } \\
\text { em diabetes }\end{array}$ & 2013 & Mestre & $\begin{array}{l}\text { Grupos de educação em } \\
\text { saúde }\end{array}$ \\
\hline 8 & $\begin{array}{c}\text { Acurácia das intervenções de enfermagem para } \\
\text { pacientes com diabetes mellitus tipo } 2 \mathrm{em} \\
\text { consulta ambulatorial }\end{array}$ & 2013 & Doutora & $\begin{array}{l}\text { Promoção da atividade física } \\
\text { e aconselhamento nutricional }\end{array}$ \\
\hline 9 & $\begin{array}{l}\text { Eficácia da educação em saúde no tratamento não } \\
\text { medicamentoso da hipertensão arterial }\end{array}$ & 2013 & $\begin{array}{c}\text { Não } \\
\text { divulgado }\end{array}$ & $\begin{array}{l}\text { Grupos de educação em } \\
\text { saúde }\end{array}$ \\
\hline 10 & $\begin{array}{l}\text { Atividades participativas em grupos de educação } \\
\text { em saúde para doentes crônicos }\end{array}$ & 2014 & Doutora & $\begin{array}{l}\text { Rodas de discussão, } \\
\text { dinâmicas, palestras, } \\
\text { caminhadas, teatro e } \\
\text { alongamento }\end{array}$ \\
\hline 11 & $\begin{array}{l}\text { Como os profissionais da atenção primária } \\
\text { percebem e desenvolvem a educação popular em } \\
\text { saúde? }\end{array}$ & 2014 & $\begin{array}{c}\text { Não } \\
\text { divulgado }\end{array}$ & Palestras em grupo \\
\hline 12 & $\begin{array}{l}\text { Contribuições de grupos de educação em saúde } \\
\text { para o saber de pessoas com hipertensão }\end{array}$ & 2014 & Especialista & Palestras educativas \\
\hline 13 & $\begin{array}{l}\text { Educação que produz saúde: atuação da } \\
\text { enfermagem em grupo de hipertensos }\end{array}$ & 2015 & Mestre & $\begin{array}{l}\text { Grupos de educação em } \\
\text { saúde }\end{array}$ \\
\hline 14 & $\begin{array}{c}\text { Avaliação de grupos de educação em saúde para } \\
\text { pessoas com doenças crônicas }\end{array}$ & 2015 & Doutora & Palestras em grupo \\
\hline 15 & $\begin{array}{c}\text { Analise de três estratégias de educação em saúde } \\
\text { para portadores de hipertensão arterial }\end{array}$ & 2016 & $\begin{array}{c}\text { Não } \\
\text { divulgado }\end{array}$ & $\begin{array}{c}\text { Oficinas educativas e visita } \\
\text { domiciliar }\end{array}$ \\
\hline
\end{tabular}

Quadro 3. Caracterização descritiva dos estudos conforme o título, ano, titulação do autor e as intervenções de enfermagem para a educação em saúde. Recife-PE, 2016

Fonte: Elaboração própria (2016). 


\section{Discussão}

ISSN 2179-6750

De acordo com os dados coletados na pesquisa, pôde-se notar que, atualmente, a prioridade nos serviços de saúde pública está direcionada aos pacientes portadores de diabetes e hipertensão.Com a finalidade de debater a importância da educação na saúde pública e suas abordagens mais usadas, fazendo uma análise comparativa entre as duas técnicas de educação e aprendizagem mais usadas, que são o método tradicional e a conscientização da população defendida por Paulo Freire (1921-1997).

A educação é um instrumento fundamental para o trabalho da enfermagem no que diz respeito à prevenção, tratamento e controle de agravos, oferecendo uma assistência de qualidade, sabe-se que o enfermeiro além de prestar o cuidado é também um educador, tanto para o paciente quanto para a família, realizando orientações e estimulando o cuidar de si. A qualidade das propostas educativas inclui a capacitação dos profissionais e das equipes de saúde a partir da perspectiva dos determinantes socioambientais que interferem no processo saúde e doença, mas, sobretudo, na adoção de um modelo dialógico que valorize os saberes da comunidade somando-os aos conhecimentos científicos e transformando-os quando necessário. ${ }^{17,18}$

Nestatabulação foi possível identificar dois tipos distintos de didática educacional que atuam no combate às doenças crônicas analisadas neste estudo tiveram maior destaque, sendo que $33,34 \%$ da amostra abordava a metodologia freireana e $40 \%$, dentre os métodos de ensino mais utilizados, foi a metodologia tradicionalista.

\section{Metodologia Freireana utilizando grupos de educação em saúde}

Baseado nos dados encontrados, 33,34\% dos artigos pesquisados abordava a metodologia Freireana como sendo a que obtinha melhores resultados. É importante salientar que, Paulo Freire destacou as falhas do modelo educacional brasileiro e, evidenciou seus defeitos por meio de estudos que expuseram a educação e suas falhas em diversas esferas da sociedade como um todo. Entre os diversos assuntos abordados em 
suas pesquisas, ele mostrou que o molde educacional abordado pelo Governo consistia basicamente na marginalização da população de baixa renda, excluindo-as da oportunidade de ter acesso à educação. ${ }^{19,20}$

Com o fim da Ditadura Militar em 1985, inúmeros movimentos sociais eclodiram em diversas áreas que pediam por uma reestruturação social e educacional. É nesse contexto de mudanças significativas que ainda na década de 80 , a metodologia pedagógica criada por Paulo Freire surgiu, como agente transformador da mudança da pedagogia educacional do país, atrelando a noção do sujeito à cidadania. ${ }^{21,22}$

O Método desenvolvido por Paulo Freire é a síntese da teorização implícita na prática de educação popular. Ela traz a importância do conhecimento como possibilidade de superação de relações verticais opostas e de modelos mecanicistas de análise da realidade social e criação de novas propostas que apontem esperança e a necessidade de mudança. Essa implementação do conhecimento deve ser enriquecida pela interdisciplinaridade, a qual é caracterizada pelo compartilhamento de diversos saberes que vão além da área da saúde e que abrangem outros setores, como, por exemplo, as sociais e as humanas. ${ }^{19,18}$

Inserindo esse contexto para o âmbito da educação na saúde pública, a didática freireana defende que haja uma interação entre os aspectos socioculturais de uma comunidade e as informações técnicas passadas pelos profissionais de saúde pública. As ações educativas nessa abordagem visam conscientizar o cliente através de ações educativas reflexivas que levam em consideração o meio ambiente inserido, dúvidas, questionamentos, necessidades. A troca de informações de modo horizontal permite o crescimento mútuo através de experiências vivenciadas por ambas às partes, com participação ativa do cliente, conteúdo programático adequado à realidade e necessidades do mesmo, predomínio do diálogo crítico. ${ }^{6,17}$

Assim, para formar uma educação inovadora, interativa e analítica, é imprescindível que se faça uma releitura no que diz respeito ao caráter ético e político dos modelos mais 
conservadores, com a finalidade de que a educação contemple a todos os cidadãos e que não haja favorecimento de classes sociais mais elevadas, fomentando assim um crescimento das discrepâncias socioeconômicas, que sedimenta o acesso a uma educação mais igualitária, mas que abrace a individualidade de cada comunidade e consequentemente sua população e se tornando fator ativo em seu próprio método. ${ }^{18,19}$

\section{Métodos tradicionais e a utilização das palestras educativas}

De acordo com os dados encontrados nessa pesquisa, foi possível evidenciar que a abordagem tradicional de educação na saúde pública corresponde a $40 \%$ dentre os métodos de ensino mais utilizados e, é uma prática formal de ensino concentrado no professor, onde o aluno apenas ouve os ensinamentos do seu tutor, e este detém a tarefa de instruí-los. Analisando através de uma expectativa macro, esse método é caracterizado por se preocupar mais com a variedade e quantidade de noções/conceitos/informações que com a formação do pensamento reflexivo. Ao cuidar e enfatizar a correção, a beleza, o formalismo, acaba reduzindo o valor dos dados sensíveis ou intuitivos, o que pode ter como consequência, a redução do ensino a um processo de impressão, a uma pura receptividade..$^{20,23,24}$

Dentro do modelo tradicional, os educadores lapidam e os educandos são lapidados, esse modelo educacional objetiva apenas em aumentar o conhecimento dos alunos, não se preocupando com o indivíduo e o seu meio. Nesse contexto, o profissional de saúde não consegue se desvincular do saber técnico, considerando este como verdade absoluta, ignorando assim a necessidade de considerar o conhecimento popular adquirido por aquele indivíduo e o ambiente social no qual se encontra inserido. ${ }^{25,26}$

Vale destacar que, a população acostumou-se com uma relação entre médicopaciente, a um caráter meramente restritivo, ou seja, restringia-se apenas a consultas médicas e acompanhamento de casos, causando assim um efeito de dependência do usuário a sempre se reportar em caráter de consulta, impedindo que o mesmo tenha 
interesse em qualquer outro tipo de abordagem, tais como, as educativas. ${ }^{4,22}$

Ao se tratar do método no qual o profissional de saúde irá utilizar para educar seus pacientes, nota-se que, muitos deles ainda estão presos aos moldes tradicionais, no qual há uma valorização do conhecimento científico. No entanto, deve-se entender que tanto os profissionais de saúde quanto os usuários são figurantes sociais que estão em constante simbiose e que se deve considerar o ambiente no qual os pacientes estão inseridos, procurando não impor práticas técnicas com rigidez, e sim procurar harmonizar com as práticas culturais de cada comunidade, inserindo o paciente num contexto de dialética e troca de conhecimento. ${ }^{1,24}$

Diante deste contexto, é possível perceber que uma das maneiras mais disseminadas sobre educação e saúde é aquela na qual as execuções se intensificam mediante condições formais de ensino aprendizagem. O processo educativo gira em torno de uma pessoa que ensina a outra pessoa que não tem conhecimento acerca do assunto abordado. Há um grande risco de o profissional tomar-se uma autoridade superior, o único responsável pelo processo de aprendizagem. A discussão que permeia as práticas da educação em saúde nos serviços atuais, especificamente nas atividades desenvolvidas na ESF, se encontra atrelada aos discursos higienistas e moralistas. Desta forma, os usuários não conseguem vislumbrar a real importância das atividades educativas em relação ao cuidado em saúde. ${ }^{27,28}$

\section{Considerações finais}

O presente estudo utilizou a abordagem bibliográfica a respeito da educação em saúde, e os tipos predominantes de abordagem no que diz respeito à educação na saúde pública no programa HIPERDIA. É importante salientar que os objetivos da pesquisa foram alcançados ao apresentar as características da produção nacional sobre as práticas de educação em saúde realizadas por enfermeiros para pacientes do programa HIPERDIA. 
De acordo com os dados coletados durante a pesquisa, notou-se que a literatura aponta uma tendência de seguir os moldes tradicionais, evidenciados no percentual de $40 \%$ com as palestras educativas, conforme consta nos artigos e periódicos pesquisados. Não há, por meio do profissional de saúde, um interesse em abordagens pedagógicas mais modernas, como por exemplo, a metodologia "freireana" que corresponde a 33,34\% da amostra, a qual aborda o uso dos grupos de educação em saúde. As implicações relacionadas à educação em saúde demonstram que há a necessidade de um melhor gerenciamento de atividades por meio dos agentes e profissionais de saúde pública, juntamente com atividades de caráter incentivador e participativo, havendo assim uma maior interação entre os cidadãos e os profissionais de saúde.

Por meio do desenvolvimento da pesquisa, foi possível concluir que a educação em saúde, apesar de toda sua variação quanto aos métodos utilizados, possui como sua essência a educação, tendo como finalidade na área de saúde a promoção de ações e esclarecimentos à população para que esta desenvolva maior autonomia no que diz respeito às informações quanto a convivência com a diabetes e a hipertensão. Desta forma, os pacientes passarão a ter uma visão mais reflexiva e elucidativa no que diz respeito às suas próprias enfermidades e limitações.

Vale ressaltar que o tema carece de maior abrangência quanto ao local de estudo, diminuindo, assim, as margens de diferença entre a população-alvo, como também outros estudos precisam ser feitos para diminuir as diferenças entre as realidades das diversas capitais brasileiras, visando obter maiores informações sobre o tema abordado nesta pesquisa.

\section{Referências}

1. Roecker S, Marcon SS. Educação em saúde na estratégia saúde da família: o significado e a práxis dos enfermeiros.Esc Anna Nery. 2011;15(4):701-9. http://dx.doi.org/10.1590/S1414-81452011000400007. 
2. Organização Mundial da Saúde. Carta de Ottawa: primeira conferência internacional sobre promoção da saúde. Ottawa: OMS, 1986.

3. Silva $C M C$, Meneghim $M C$, Pereira AC, Mialhe FL. Educação em saúde: uma reflexão histórica de suas práticas. Cienc Saude Colet. 2010;15(5):2539-50.

http://dx.doi.org/10.1590/S1413-81232010000500028.

4. David HMSL, Acioli S. Mudanças na formação e no trabalho de enfermagem: uma perspectiva da educação popular e de saúde. Rev Bras Enferm. 2010;63(1):127-31. http://dx.doi.org/10.1590/S0034-71672010000100021.

5. Berbel DB, Rigolin CCD. Educação e promoção da saúde no Brasil através de campanhas públicas. Ver Bras Cienc Tecnol Soc. 2011 [citado4 out 2016];2(1):25-38. Disponível em: http://vianabarmann.com.br/wp-content/uploads/2014/08/124-465-1-PB.pdf.

6. Nogueira IS, Vergaças HM, Santos LF, Cypriano PE, Moreno MG, Lima SO, et al. A práticaeducativana estratégia saúde da família: estratégia para repensar e reconstruir ações dialógicas. Arq Cienc Saude UNIPAR. 2015;19(1):11-7. https://doi.org/10.25110/arqsaude.v19i1.2015.5259.

7. Lima AS, Gaia ESM, Ferreira MA. Aimportância do programaHiperdia em uma unidade de saúde da família do município de Serra Talhada - PE, para adesão dos hipertensos e diabéticos ao tratamento medicamentoso e dietético. Saude Colet Deb. 2012[4 out 2016];2(1):9-17. Disponível em: http://fis.edu.br/revistaenfermagem/artigos/vol02/artigo02.pdf.

8. Matsumoto PM, Barreto ARB, Sakata KN, Siqueira YMC, Zoboli ELCP, Fracolli LA. A educação em saúde no cuidado de usuários do Programa Automonitoramento Glicêmico. Rev Esc Enferm USP. 2012;46(3):761-5. http://dx.doi.org/10.1590/S008062342012000300031.

9. Reticena KO, Piolli KC, Carreira L, Marcon SS, Sales CA. Perce pção de idososacerca das atividades desenvolvidas no hiperdia. Rev Min Enferm. 2015;19(2):107-13. http://dx.doi.org/10.5935/1415-2762.20150029.

10. Ministério da Saúde (BR). Organização Pan-Americana da Saúde. Avaliação do plano de reorganização da atenção à hipertensão arterial e ao diabetes mellitus no Brasil. Brasília, DF: OPAS, 2004 [citado10 out 2016]. Disponível em: http://bvsms.saude.gov.br/bvs/publicacoes/diab.pdf. 
11. Lima JC, Santos AL, Marcon SS. Percepção de usuários com hipertensão acerca da assistência recebida na atenção primária. Rev Pesqui Cuid Fundam Online. 2016;8(1):3945-56. http://dx.doi.org/10.9789/2175-5361.2016.v8i1.3945-3956.

12. Carvalho Filha FSS, Nogueira LT, Viana LMM. Hiperdia: adesão e percepção de usuários acompanhados pela estratégia saúde da família. Rev Rene. 2011;12(Esp):930-6. http://dx.doi.org/10.15253/rev\%20rene.v12i0.4380.

13. Brasil. Ministério da Saúde. Secretaria de Atenção à Saúde. Departamento de Atenção Básica. Estratégias para o cuidado da pessoa com doença crônica: hipertensão arterial estrasistêmica. Brasília, DF: SAS/DAB; 2013 [citado10 out 2016]. 128 p. Disponível em: http://bvsms.saude.gov.br/bvs/publicacoes/estrategias_cuidado_pessoa_doenca_cronica. pdf.

14. Jorge JC, Guimarães CM. Estratégia saúde da família: a enfermagem e o cuidar humanizado. Rev Estudos. 2014;41(Esp):113-24. Disponível em: http://seer.ucg.br/index.php/estudos/article/viewFile/3812/2176.

15. Marconi MAL. Técnicas de pesquisa: planejamento e execução de pesquisas, amostragens e técnicas de pesquisas, elaboração, análise e interpretação de dados. 7 a ed. São Paulo: Atlas; 2011.

16. Whittemore R, Knafl K. The integrative review: updated methodology. J Adv Nurs. 2005;52(5):546-53. http://dx.doi.org/10.1111/j.1365-2648.2005.03621.x.

17. Lanzoni GMM, Meirelles BHS. Liderança do enfermeiro: uma revisão integrativa da literatura. Rev Latino-Am Enferm. 2011;19(3):651-8. http://dx.doi.org/10.1590/S010411692011000300026.

18. Conrado DM, Nunes-Neto NF, El-Hani CN. Aprendizagem baseada em problemas (ABP) na educação científica como estratégia para formação do cidadão socioambientalmente responsável. Rev Bras Pesq Educ Cienc. 2014 [citado17 nov 2016];14(2):77-87. Disponível em: https://seer.ufmg.br/index.php/rbpec/article/viewFile/2692/2058.

19. L'Abbate S. Educação em saúde: uma nova abordagem. Cad Saude Publica. 1994;10(4):481-90. Disponível em: http://dx.doi.org/10.1590/S0102$311 \times 1994000400008$.

20. Falkenberg MB, Mendes TPL, Moraes EP, Souza EM. Educação em saúde e educação na saúde: conceitos e implicações para a saúde coletiva. Cienc Saude Colet. 2014;19(3):84752. http://dx.doi.org/10.1590/1413-81232014193.01572013. 
21. Levy N. Desejo... O Lugar da Liberdade: um ensaio ético-político. São Paulo: Debates; 1991.

22. Freire P. Cartas à Guiné-Bissau: registros de uma experiência em processo. Rio de Janeiro: Paz e Terra; 1978.

23. Sousa JDL, Zveiter M, Almeida VLMD, Menezes HFD, Mara G, Alves R. Educação em saúde como ferramenta à mulher no climatério: subsídios para o cuidado de enfermagem. Rev Pesq Cuid Fundam Online. 2012 [citado 20 nov 2016];4(1):2616-22. Disponível em: http://www.seer.unirio.br/index.php/cuidadofundamental/article/view/1485/pdf_471.

24. Gomes LB, Merhy EE. Compreendendo a educação popular em saúde: um estudo na literatura brasileira. Cad Saude Publica. 2011;27(1):7-18. http://dx.doi.org/10.1590/S0102-311X2011000100002.

25. Sá LD, Gomes ALC, Carmo JB, Souza KMJ, Palha PF, Alves RS, Andrade SLE. Educação em saúde no controle da tuberculose: perspectiva de profissionais da estratégia saúde da família. Rev Eletr Enferm. 2013;15(1),103-11. Disponível em: http://dx.doi.org/10.5216/ree.v15i1.15246.

26. Freire P. Pedagogia do oprimido. 46a ed. Rio de Janeiro: Paz e Terra; 2005.

27. Flisch TMP, Alves RH, Almeida TAC, Torres HC, Schall VT, Reis DC. Como os profissionais da atenção primária percebem e desenvolvem a Educação Popular em Saúde?. Interface. 2014;18(Supl. 2):1255-68. http://dx.doi.org/10.1590/1807-57622013.0344.

28. Ferreira ADS, Costa EFL, Farias KF, Bezerra RP, Dantas TF, Zacarias VS. A história da educação em saúde e seus modelos de prática impostos à sociedade. Diversitas J. 2016;1(1):48-54. Disponível em: http://dx.doi.org/10.17648/diversitas-journal-v1i1.379.

29. Jacomé PS, Araujo JL, Pereira AKAM, Vasconselos RB, Nascimento EGC. A educação em saúde realizada por profissionais da saúde da estratégia saúde da família. RUVRV. 2016;14(1):160-77. Disponível em: http://dx.doi.org/10.5892/ruvrd.v14i1.2450. 


\section{Minicurrículo}

Sílvio Nicolau| ORCiD: 0000-0002-0123-208X

Mestre em Enfermagem pela Faculdade de Enfermagem Nossa Senhora das Graças - FENSG. Enfermeiro do Hospital da Restauração - HR, em Pernambuco. Docente da FAREC, Recife. Docente como Prof. Substituto do Departamento de Enfermagem da Universidade Federal de Pernambuco - UFPE, na área de Enfermagem Médico-Cirúrgico.

Kely Joelma Dantas de Almeida| ORCiD: 0000-0002-0123-208X

Graduanda do curso de Enfermagem da Faculdade Mauricio de Nassau - UNINASSAU, Recife, PE, Brasil.

Adriana Moura| ORCiD: 0000-0002-0123-208X

Graduanda do curso de Enfermagem da Faculdade Mauricio de Nassau - UNINASSAU, Recife, PE, Brasil.

Jackeline Simas| ORCiD: 0000-0002-0123-208X

Graduanda do curso de Enfermagem da Faculdade Mauricio de Nassau - UNINASSAU, Recife, PE, Brasil. 